\title{
Electron cooling and finite potential drop in a magnetized plasma expansion
}

\author{
M. Martinez-Sanchez, ${ }^{1}$ J. Navarro-Cavallé, ${ }^{2}$ and E. Ahedo ${ }^{3}$ \\ ${ }^{1}$ Department of Aeronautics and Astronautics, Massachusetts Institute of Technology, \\ 77 Massachusetts Avenue, Cambridge, Massachusetts 02139, USA \\ ${ }^{2}$ Escuela Técnica Superior de Ingeniería Aeronáutica y del Espacio, Universidad Politécnica de Madrid, \\ Plaza Cardenal Cisneros 3, Madrid 28040, Spain \\ ${ }^{3}$ Departamento de Bioingeniería e Ingeniería Aeroespacial, Universidad Carlos III de Madrid, Avda. \\ Universidad 30, Leganés 28911, Madrid, Spain
}

(Received 30 January 2015; accepted 21 April 2015; published online 5 May 2015)

The steady, collisionless, slender flow of a magnetized plasma into a surrounding vacuum is considered. The ion component is modeled as mono-energetic, while electrons are assumed Maxwellian upstream. The magnetic field has a convergent-divergent geometry, and attention is restricted to its paraxial region, so that $2 \mathrm{D}$ and drift effects are ignored. By using the conservation of energy and magnetic moment of particles and the quasi-neutrality condition, the ambipolar electric field and the distribution functions of both species are calculated self-consistently, paying attention to the existence of effective potential barriers associated to magnetic mirroring. The solution is used to find the total potential drop for a set of upstream conditions, plus the axial evolution of various moments of interest (density, temperatures, and heat fluxes). The results illuminate the behavior of magnetic nozzles, plasma jets, and other configurations of interest, showing, in particular, in the divergent plasma the collisionless cooling of electrons, and the generation of collisionless electron heat fluxes. (2015 AIP Publishing LLC.

[http://dx.doi.org/10.1063/1.4919627]

\section{INTRODUCTION}

The dynamics of steady or transient plasma expansions has long attracted attention due to its fundamental importance for many applications. Despite its relative simplicity, the problem involves certain conceptual difficulties, particularly in explaining the mechanism by which electrons canagainst the potential field gradient-follow the accelerated ions in a vacuum plasma expansion. Despite early feasibility concems when Ion Engines were first developed, ${ }^{1,2}$ expansions of this sort are now routine in many fields. With the advent of powerful simulation tools, modelers have been able to show that indeed, a steady collision-free expansion is possible, ${ }^{3}$ but by their nature, these simulations do not fully disclose the mechanisms that are involved. They do, however, illustrate the importance of a trapped electron population that develops during the start-up transient.

Among the basic persistent difficulties, there is the fact that simple closures of the fluid equation hierarchy have failed to produce a model for the heat fluxes in the absence of collisions, so that one is forced to adopt ad-hoc electron temperature models, like isothermal, polytropic, or adiabatic. The isothermal assumption is attractive because, aside from its simplicity that leads to the Boltzmann relationship between density and potential, it is justifiable if the distribution remains close to Maxwellian. However, it is easy to show that an isothermal electron population, being a local inexhaustible source of thermal energy, leads to unbounded acceleration of the plasma. For the same reason, an isothermal model fails to predict a finite total potential drop along the plume, even though only a finite amount of thermal energy is initially available. Preliminary analyses of ion and electron thermodynamics effects on the plasma expansion through a magnetic nozzle
(MN) were investigated by Merino and Ahedo. ${ }^{4}$ It seems physically clear that the electron and ion temperatures must eventually drop to zero or near zero, but a simple adiabatic law cannot be kinetically justified, and no rigorous alternative has emerged. Aside from the intrinsic interest of these questions, knowledge of the electron temperature to be expected far downstream from the source is technologically important if this distant plasma is to be in contact with sensitive surfaces.

Liemohn and Khazanov ${ }^{5}$ provide a review of the extensive Astrophysics literature related to this problem. These authors also present a model that is, in some respects, similar to ours, but does not impose quasi-neutrality. Arefiev and Breizman ${ }^{6}$ also consider this problem, including quasi-neutrality, via an effective potential for paraxial electron motion, which is an equivalent alternative to the approach employed here. They discuss the existence of a "decoupled" electron population, cut off by a magnetically induced potential energy barrier. This appears to be a reference to a trapped electron population, which is a possibility we also consider, however, the approach of Ref. 6 to this population is obscure, invoking a downstream "expansion wave," apparently unsteady, that somehow connects to the steady near-throat flow, and which is said to add momentum to the plasma even past its full expansion.

In this paper, we consider what is possibly the simplest model capable of providing rigorous answers to these questions. The basic assumptions are: (a) magnetized electrons and ions; (b) slender geometry, basically that of one magnetic streamtube; (c) magnetic field profile starting and ending at zero intensity, with a peak that defines the throat; (d) Maxwellian electrons at the source, with the steady spatial distribution being one of the results of the calculation; (e) existence of a population of trapped electrons in some portion 
of the energy-magnetic moment map; (f) mono-energetic ions, isotropic at the source; (g) no ionizing or scattering collisions; (h) quasi-neutrality throughout; and (i) zero net current, or a prescribed net current.

The neutrality condition is satisfied by a unique selfconsistent plasma potential profile along the nozzle. More specifically, the magnetic field is found to have a unique relationship to the plasma potential, so that all other quantities can be expressed as functions of the local potential, with no reference to the particular dependence of that potential on axial distance. The finite total potential drop between the two magnetic field end points is one of the important results of the model; it depends on the initial electron temperature, and it defines the asymptotic ion velocity, which, in the propulsion context, gives the specific impulse of the device. Since the electron and ion distribution functions are computed for each location, all moments are directly calculable as well. This includes the perpendicular and parallel temperatures of both species, and the electron heat flux, with its two contributions due to random transport of perpendicular and parallel random energy.

This work is motivated by the current research work in some space plasma thrusters that use a MN to guide and accelerate the plasma jet. These electrodeless, current-free thrusters (in contrast to the mature Ion and Hall thrusters that use an external cathode to neutralize the ion beam) differ from each other basically on the plasma production stage. For instance, the helicon plasma thruster, which has been broadly developed in different institutions during the last decade, ${ }^{7-10}$ uses a helicon source to produce and heat the plasma, which is then accelerated in a $\mathrm{MN} .{ }^{11}$ The VASIMR $^{12,13}$ uses a helicon source to produce the plasma, an Ion Cyclotron Resonance stage to heat it, and a $\mathrm{MN}$ to finally accelerate the hot plasma beam. A last example is the ECR (Electron Cyclotron Resonance) plasma thruster, ${ }^{14}$ which combines the use of an ECR plasma source and a MN.

The paper is organized as follows: Section II is devoted to the formulation of the kinetic model, leading to an integral equation for the potential distribution as a function of the magnetic field distribution. In Sec. III, we explain the iterative method of solution of this equation. The results are presented in Sec. IV, where the various moments of the electron and ion distributions are calculated from their distribution functions, and parametric sweeps are shown for the initial electron and ion temperatures, the ion-to-electron mass ratio, and the overall current carried by the jet. In Sec. V, we take a critical look at the assumptions and discuss the limitations and potential extensions of the method.

\section{FORMULATION}

In this section, the formulation of the model is summarized. We split it into Subsections II A-II D, each one describing ion and electron densities, and the neutrality and current conditions.

\section{A. The ion density}

The general approach, and much of the notation, is similar to that used in Ref. 15 for the related problem of a magnetic cusp. In that work, the electron population was fairly well confined (by both, the electrostatic and magnetic fields), and the small fraction absorbed by the wall was neglected, leading to an isothermal and isotropic Maxwellian electron distribution. In contrast, the ions, although still magnetically confined, were accelerated by the pre-sheath potential drop, and their distribution function was to be found. Two cases were considered: one with a mono-energetic, isotropic ion distribution at the source and one with a Maxwellian ion distribution at the source. It was found that the main results were quite similar in both cases, with the exception of the ion heat flux, whose parallel energy part was zero in the mono-energetic case, but non-zero in the Maxwellian case. For application to our convergent-divergent geometry, we notice in addition that, regardless of the distribution at the source, the ion acceleration implies a gradual approach to a beam-like distribution, close to a single energy as well. We therefore adopt for the ions, a simple mono-energetic source distribution function

$$
f_{i}=\frac{m_{i} n_{\infty}}{4 \pi}\left(\frac{m_{i}}{2 E_{i \infty}}\right)^{1 / 2} \delta\left(E_{i}-E_{i \infty \infty}\right),
$$

with nommalization $\iiint f_{i} d^{\beta} w=n_{\infty}$ is the plasma density at the reservoir, $m_{i}$ is the ion mass, $\delta$ is the Dirac delta and $E_{i}$ the ion energy (mono-energetic here, and defined at the reservoir $\left.E_{i \infty}\right)$. The equivalent ion temperature at the source is $T_{\infty}=(2 / 3)\left(E_{\infty} / k\right)$, with $k$ the Boltzmann constant. Hereafter, subscripts $e, i$, will refer to a property evaluated for electrons and ions respectively, and $\infty$ will refer to a property given at the upstream reservoir. The magnetic moment of a given ion is conserved along its trajectory $\left(\mu_{i}=m_{i} w_{\perp}^{2} / 2 B=\right.$ const), and so its (conserved) energy can be written as $E_{i}=m_{i} w_{\|}^{2} / 2+\mu_{i} B+e \phi$, where $w_{\|}$is the velocity component along $\mathbf{B}, w_{\perp}$ is the velocity component perpendicular to $\mathbf{B}$, and $\phi$ is the electrostatic potential at the point where the magnetic field is B. In what follows, we will take the source potential to be zero, so that $\phi$ is generally negative. For a fixed $E_{i}$, the maximum magnetic moment at a point is then

$$
\mu_{i m}=\frac{E_{i}-e \phi}{B} .
$$

This quantity has a minimum value at some point, in the convergent portion of the magnetic channel, where $\phi$ is falling and $B$ is increasing. We designate this minimum as

$$
\mu_{i T}\left(E_{i}\right)=\min \left\{\mu_{i m}\left(B, E_{i}\right)\right\} \text { at } B=B_{i T}\left(E_{i}\right) .
$$

Hereafter, all properties evaluated at that point are labeled with the T subscript. Downstream of where $B_{i T}$ occurs, only ions with $\mu_{i}<\mu_{i T}$ exist. Upstream of $B_{i T}$, there are ions with $\mu_{i T}<\mu_{i}<\mu_{i m}$ moving with $w_{\|}>0$, as well as those with the same magnetic moment that have been bounced back by the magnetic mirror moving with $w_{\|}<0$. The full distribution function is therefore a spherical segment shell in velocity space, determined by the angle $\theta_{i}$, as depicted in Figure 1.

For these mono-energetic ions, the average or mean value of any quantity $\chi_{i}$ is then 


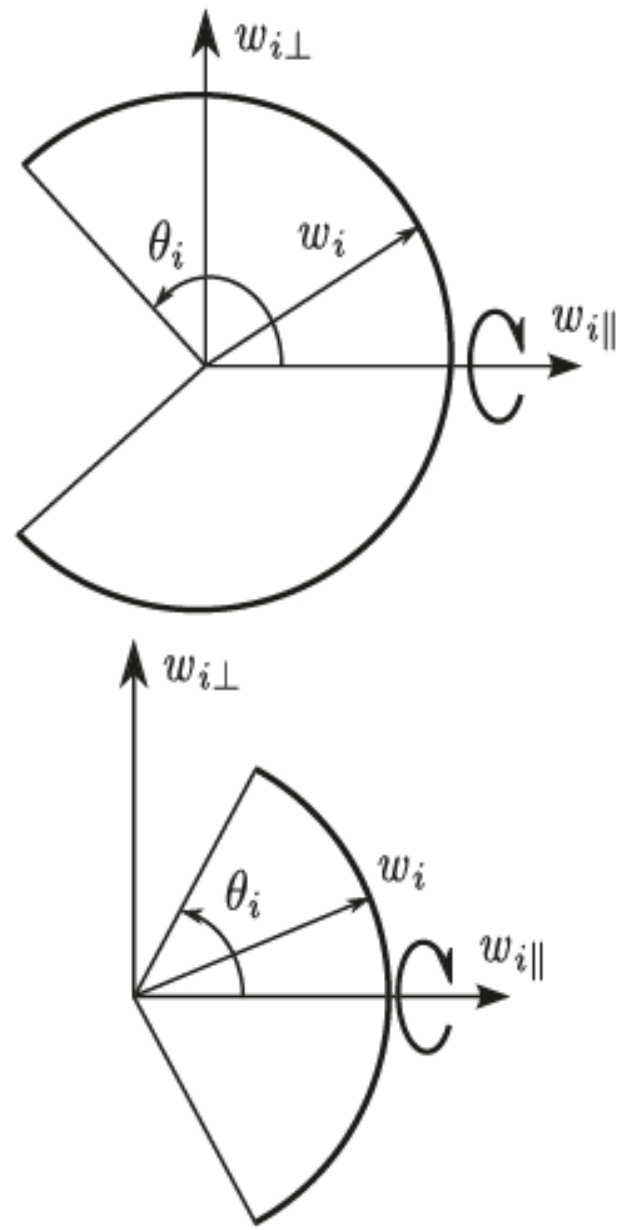

FIG. 1. Ion distribution functions for the regions to the left and to the right of where $B=B_{a r}$ (top figure, bottom figure). Here, $\theta_{i}=\sin ^{-1} \sqrt{\mu_{i T} / \mu_{i m}}$, and $w=\sqrt{2\left(\bar{E}_{i}-e \phi\right) / m_{i}}, \theta_{i}=\theta_{i T}=\pi / 2$ for $\mu_{i m}=\mu_{a T}$ at $B=B_{i T}$.

$$
\begin{aligned}
\left\langle\chi_{i}\right\rangle= & \frac{n_{\infty}}{n_{i}} \frac{1}{4 \pi}\left(\frac{m_{i}}{2 E_{i \infty}}\right)^{1 / 2} \int_{0}^{\infty} \int_{0}^{\theta_{i}\left(E_{i}\right)} \chi_{i} \delta\left(E_{i}-E_{i \infty}\right) \\
& \times 2 \pi w_{i} \sin \theta_{i} d \theta_{i} d E_{i} .
\end{aligned}
$$

In particular, for $\chi_{d}=1$, we obtain the ion density

$$
\begin{aligned}
n_{i} & =\frac{n_{\infty}}{2}\left(\sqrt{1-\frac{e \phi}{E_{i}}} \pm \sqrt{1-\frac{e \phi+\mu_{i T} B}{E_{i}}}\right) \\
& =\frac{n_{\infty} \sqrt{B}}{2 \sqrt{E_{i}}}\left(\sqrt{\mu_{i m}} \pm \sqrt{\mu_{i m}-\mu_{i T}}\right),
\end{aligned}
$$

where the upper sign applies upstream of where $B=B_{i T}$, and vice versa. This analysis is identical to that in Ref. 15 , except that it now extends to the divergent $B$-field side as well.

\section{B. The electron density}

The formulation is, in principle, similar for electrons of a particular energy $E_{e}$, but important new effects occur when the sign of the charge is reversed. As for ions, there is a maximum electron magnetic moment at the location where the field is $B$ and the potential is $\phi$, namely,

$$
\mu_{e m}=\frac{E_{e}+e \phi}{B} \text {. }
$$

But now the numerator is everywhere decreasing in the flow direction, and so a minimum $\mu_{e T}\left(E_{e}\right)$ of $\mu_{e m}$-if it existsmust happen where $B$ is also decreasing, i.e., in the diverging field section. In addition, unlike the ion case, the numerator of Eq. (6) may cross zero before the end of the channel. At a location where the potential is $\phi$, electrons with $E_{e}<-e \phi$ must have been electrostatically bounced back upstream, and this low energy range must be absent from the distribution, or, equivalently, $\mu_{\mathrm{em}}$ must be positive.

The behavior of the electron distribution function can be understood with reference to Figure 2, which advances some of our numerical results.

For any given energy, no electrons can be present above the corresponding $\mu_{e m}$ line in Figure 2, as this would imply a negative parallel kinetic energy. Also, as noted above, $\mu_{\mathrm{em}}$ crosses through zero where $E_{e}=-e \phi$, or $\varepsilon_{e}=\Phi$.

As Figure 2 also shows, the maximum magnetic moment $\mu_{e m}$ is monotonically decreasing for low enough energies, roughly $\varepsilon_{e}<\varepsilon_{e}^{*}=0.49$ in this case. Since all electrons have constant positive magnetic moments, this means that electrons below this energy will be bounced back, and the distribution will be a complete spherical shell for all these energies below the line $\varepsilon_{e}=\varepsilon_{e}^{*}$.

For energies between $\varepsilon_{e}^{*}$ and unity (corresponding to electrons that are just able to escape the nozzle), we can see in Figure 2 the existence of a minimum and a maximum of $\mu_{e m}$. For these, the minimum, $\mu_{e T}$, plays a similar role as $\mu_{i T}$ does for ions: any electron with $\varepsilon_{e}>\varepsilon_{e}^{*}$ and $\mu_{e}>\mu_{e T}$ will be bounced back upstream of the location where this minimum happens. Electrons with $\mu_{e}<\mu_{e T}$ can pass through this choke point, but will be bounced back as they reach the location where their magnetic moment equals the local $\mu_{e m}$, past the maximum of the $\mu_{e m}$ curve. Since the higher magnetic moments have been excluded, these electrons have a distribution in the shape of two symmetric spherical segments, one limited by the angle $\theta_{e}=\sin ^{-1} \sqrt{\mu_{e T} / \mu_{e m}}$ and the other by its supplementary angle $\pi-\theta_{e}$.

For energies high enough to overcome the total potential drop $\left(E_{e}>-e \phi_{\text {oud }}\right.$, or $\left.\varepsilon_{e}>1\right)$, the $\mu_{e m}$ curve does not have a

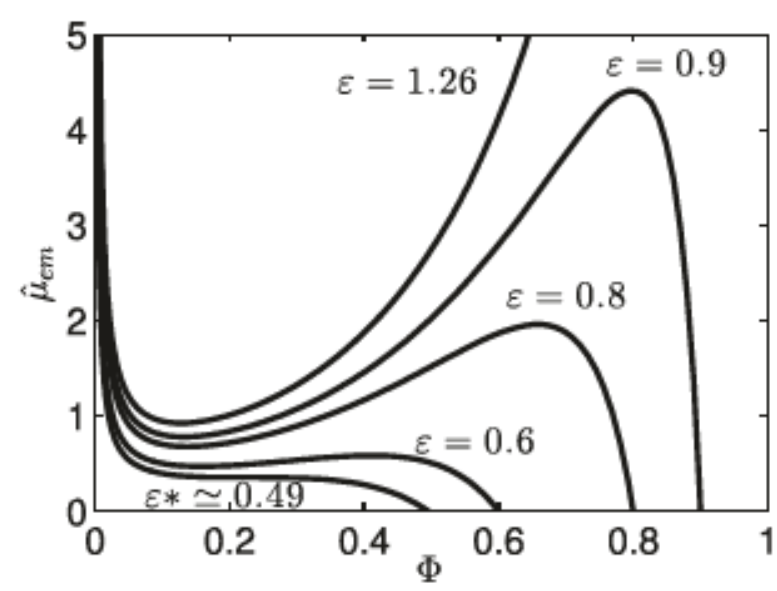

FIG. 2. The maximum local magnetic moment, $\hat{\mu}_{a m}$, versus potential drop, $\Phi$, for various electron energies, $\varepsilon_{c}$. Energy and potential are normalized by $-e \phi_{\text {ad }}$, magnetic moment by $-e \phi_{\text {out }} / B_{\max }$. These results are for $\varepsilon_{i}=$ $E_{i} / k T_{e \infty}=0.1$ and $m_{i} / m_{e}=10^{4}$. 
maximum, but diverges to infinity as the exit is approached. As a consequence, electrons with these energies that clear the barrier by having $\mu_{e}<\mu_{e T}$ can escape into the far plume and provide the neutralization for the accelerated ions.

The preceding discussion implies that those electrons with less than escape energy $\left(\varepsilon_{e}<1\right)$ and with magnetic moment between the local maximum $\mu_{e m}$ and its minimum upstream value $\mu_{e T}$, i.e., those in the dome under one of the $\mu_{e m}$ curves in Figure 2, should simply be absent from the population. This is true if their only source is the upstream plasma, from which they are isolated by the magnetic mirror barrier. However, these electrons, if present, would be also isolated (electrostatically) from the downstream environment, and this raises the possibility for a trapped population. Why this trapping might happen is a complex question that would require a rigorous analysis of the unsteady start-up process and of the possible trapping mechanisms, including collisions. One potential scenario, supported by the numerical results of Ref. 3, is as follows: Assuming the $B$ field is pre-established and that plasma is suddenly introduced upstream and allowed to expand to vacuum, one would first see electrons, with their higher random speeds, streaming out. This would produce a strongly negative potential past the throat, until at a later time the accelerated ions would arrive and start the neutralization process. As the eventual quasi-neutrality is approached, the transient local potential increase would reduce the total energy of electrons and trap those that end up below the rim of the potential energy trough that is equivalent to the dome in the diagram of Figure 2 (the potential energy invoked here is that for the effective axial motion, namely, $U=-e \phi+\mu_{e} B$ ).

The implications of the preceding discussion are summarized in the diagrams in the Appendix.

In our work, we have, in fact, made the trapping assumption. As a consequence, as the Appendix makes clear, for any non-escaping energy $\left(E_{e}<-e \phi_{\text {out }}\right)$, the angular distribution is a full spherical shell. These electrons do not contribute to any odd moment of the distribution, like the mean axial velocity. For escaping energies, the angular distribution mimics that for ions in Figure 1: a spherical shell missing a backwards segment for locations past where $\mu_{e T}$ occurs, and only the forward segment past this point.

Vlasov's equation states that the distribution function is conserved along particle trajectories, and so it must be a function of the constants of the motion. There are two such constants in our case, $E_{\mathrm{e}}$ and $\mu_{\mathrm{e}}$, and since at the source, the distribution function is Maxwellian and depends only on $E_{\mathrm{e}}$, this same dependence must apply everywhere, provided the local conditions can be connected to the source by a continuous trajectory. Thus the electrons distribution function is written as,

$$
g\left(E_{e}\right)=n_{\infty}\left(\frac{m_{e}}{2 \pi k T_{e \infty}}\right)^{3 / 2} \exp \left(-\frac{E_{e}}{k T_{e \infty}}\right) .
$$

$T_{e \infty}$ is the electron temperature defined at the source. For electrons, the moment of a generic quantity $\chi_{e}$ is then

$$
\begin{aligned}
\left\langle\chi_{e}\right\rangle= & \frac{n_{\infty}}{n_{e}}\left(\frac{m_{e}}{2 \pi k T_{e \infty}}\right)^{3 / 2} \int_{0}^{\infty} \int_{0}^{\varepsilon_{e}\left(\varepsilon_{e}\right)} \chi_{e} \exp \left(-\frac{E_{e}}{k T_{e \infty}}\right) \\
& \times 2 \pi w_{e} \sin \theta_{e} d \theta_{e} \frac{d E_{e}}{m_{e}} .
\end{aligned}
$$

Taking $\chi_{e}=1$, the local electron density at a location where the potential is $\phi$ and the magnetic field is $B$ can now be written down after performing the angular integration as

$$
\begin{aligned}
n_{e}= & \frac{n_{\infty}}{\sqrt{\pi}\left(k T_{e \infty}\right)^{3 / 2}}\left[2 \int_{-e \phi}^{-e \phi_{o \alpha}} \sqrt{E_{e}+e \phi} \exp \left(-\frac{E_{e}}{k T_{e \infty}}\right) d E_{e}\right. \\
& +\int_{-e \phi_{\infty e x}}^{\infty}\left(\sqrt{E_{e}+e \phi} \pm \sqrt{E_{e}+e \phi-\mu_{e T} B}\right) \\
& \left.\times \exp \left(-\frac{E_{e}}{k T_{e \infty}}\right) d E_{e}\right]
\end{aligned}
$$

where the factor of two in the first line (energies below escape) accounts for the two possible signs of the parallel velocity for each energy. Subscript "out" refers to a property evaluated far downstream, at the end of the expansion, say $\phi \rightarrow \phi_{\text {our }}$. In the second line, the positive sign applies upstream of $B_{e T}\left(E_{e}\right)$, and vice-versa. The integration covering the non-escaping range does not involve $\mu_{e T}\left(E_{e}\right)$, and can therefore be performed analytically, so that (9) can be rewritten as

$$
\begin{aligned}
\frac{n_{e}}{n_{\infty}}= & \exp \left(\frac{e \phi}{k T_{e \infty}}\right) e r f \sqrt{\frac{e\left(\phi-\phi_{\text {out }}\right)}{k T_{e \infty}}}-\frac{2}{\sqrt{\pi}} \exp \left(\frac{e \phi_{\text {out }}}{k T_{e \infty}}\right) \\
& \times \sqrt{\frac{e\left(\phi-\phi_{\text {out }}\right)}{k T_{e \infty}}}+\frac{1}{\sqrt{\pi}\left(k T_{e \infty}\right)^{3 / 2}} \\
& \times \int_{-e \phi_{\text {ext }}}^{\infty}\left(\sqrt{E_{e}+e \phi} \pm \sqrt{E_{e}+e \phi-\mu_{e T} B}\right) \\
& \times \exp \left(-\frac{E_{e}}{k T_{e \infty}}\right) d E_{e} .
\end{aligned}
$$

As in the case of a cusp with Maxwellian ions, treated in Ref. 15 , the difficulty that arises in evaluating these integrals is that the dependence of $\mu_{e T}$ on $E_{e}$ is not known a-priori but depends on the global solution of $\phi(B)$. We return to this point in Sec. III.

\section{The neutrality equation}

Considering Eq. (5) together with Eq. (10), we obtain the equation that relates $\phi$ to $B$. For convenience, we nommalize variables as follows:

$$
\begin{gathered}
\beta=\frac{B}{B_{\max }}, \Phi=\frac{\phi}{\phi_{\text {oud }}}, \varepsilon_{i}=\frac{E_{i}}{k T_{e \infty}}, \varepsilon_{e}=\frac{E_{e}}{\left|e \phi_{\text {out }}\right|}, \\
\hat{\mu}_{i}=\frac{\mu_{i} B_{\max }}{\left|e \phi_{\text {oud }}\right|}, \hat{\mu}_{e}=\frac{\mu_{e} B_{\max }}{\left|e \phi_{\text {out }}\right|}, \Psi=\frac{\left|e \phi_{\text {out }}\right|}{k T_{e \infty}} .
\end{gathered}
$$

According to this normalization, $\Phi$ ranges from 0 (far upstream) to 1 (far downstream) and $\Psi$-the dimensionless total potential drop-is a parameter to be determined. The neutrality condition can now be written in non-dimensional form as 


$$
\begin{aligned}
& \sqrt{1+\frac{\Psi}{\varepsilon_{i}} \Phi \pm \sqrt{1+\frac{\Psi}{\varepsilon_{i}} \Phi-\frac{\Psi}{\varepsilon_{i}} \hat{\mu}_{i T} \beta}}=2 \exp (-\Psi \Phi) \\
& \times \operatorname{erf} \sqrt{\Psi(1-\Phi)}-\frac{4}{\sqrt{\pi}} \exp (-\Psi) \sqrt{\Psi(1-\Phi)} \\
& +\frac{2 \Psi^{3 / 2}}{\sqrt{\pi}} \int_{1}^{\infty}\left(\sqrt{\varepsilon_{e}-\Phi} \pm \sqrt{\varepsilon_{e}-\Phi-\hat{\mu}_{e T} \beta}\right) \exp \left(-\Psi \varepsilon_{e}\right) d \varepsilon_{e}
\end{aligned}
$$

Aside from the double-valued function $\Phi(\beta)$, there are several parameters in this equation that need to be determined for a valid solution:

(a) The parameter $\hat{\mu}_{i T}$, which satisfies Eq. (3). This guarantees that the second radicand on the ion side of Eq. (12) is non-negative. The change of sign is made at the single point where this radicand reaches its minimum zero value, namely, at $\beta_{t T}$, somewhere along the converging field side.

(b) The function $\hat{\mu}_{e T}\left(\varepsilon_{e}\right)$, for electrons with $\varepsilon_{e}>1$. This must be such as to satisfy the definition $\hat{\mu}_{e T}\left(\varepsilon_{e}\right)$ $=\min \left\{\hat{\mu}_{e m}\left(\varepsilon_{e}, \Phi\right)\right\}$, namely, $\hat{\mu}_{e T}\left(\varepsilon_{e}\right)=\min \left\{\left(\varepsilon_{e}-\Phi\right)\right.$ $/ \beta$ \}. It can be seen from Eq. (12) that this guarantees for all $\varepsilon_{e}$ the non-negativity of the radicand inside the $\varepsilon_{e}$-integral. At the location $\beta_{e T}$ (on the diverging field side), the radicand takes its minimum value of zero at the energy $\varepsilon_{e}=\varepsilon_{e} T$.

(c) The parameter $\Psi$, which-in analogy to the potential drop through a sheath-determines the ratio of total ion to electron current.

\section{The current condition}

Since total current is a free parameter, ion and electron fluxes must be computed separately and combined to yield the given net current. Dimensionless momenta are defined and computed in Sec. IV, but ion and electron flux expressions, in particular, are advanced here, since they are invoked in the discussion of the numerical method in Sec. III

$$
\begin{aligned}
& \hat{\Gamma}_{i}=\frac{\Psi}{\sqrt{8 \varepsilon_{i}}} \beta \hat{\mu}_{i T} ; \\
& \hat{\Gamma}_{e}=\left(\frac{m_{i}}{2 \pi m_{e}}\right)^{1 / 2} \Psi^{2} \beta \int_{1}^{\infty} \hat{\mu}_{e T} \exp \left(-\Psi \varepsilon_{e}\right) d \varepsilon_{e} .
\end{aligned}
$$

The quantity $n_{\infty} c_{s}$, with $c_{s}=\sqrt{k T_{e \infty} / m_{i}}$, has been taken as the flux reference to normalize both ion and electron fluxes, $\hat{\Gamma}_{i}$ and $\hat{\Gamma}_{e}$. The net current is proportional to the difference $\hat{\Gamma}=\hat{\Gamma}_{i}-\hat{\Gamma}_{e}$. Current-free plasma will be assumed hereafter in this work, $\hat{\Gamma}=0$.

\section{METHOD OF SOLUTION}

The basic scheme used to solve Eq. (12) is:

(a) Discretize the magnetic field $\beta$ between 0 and 1 .

(b) Guess a value of the overall potential drop $\Psi$.

(c) Postulate a double-valued potential drop distribution $\Phi(\beta)$. Since $\beta$ has its maximum at some value of $\Phi$, the function $\Phi(\beta)$ should start at $\Phi=0$ for $\beta \rightarrow 0$ (far upstream), turn back at $\beta=1$, and then continue rising to $\Phi=1$ when $\beta$ approaches zero again (far downstream).

(d) Calculate the function $\hat{\mu}_{i m}=\left(\varepsilon_{i} / \Psi+\Phi\right) / \beta$ and find its minimum value $\hat{\mu}_{i T}$.

(e) For each of a number of electron energies within the range $1<\varepsilon_{e}<\infty$, calculate $\hat{\mu}_{e m}=\left(\varepsilon_{e}-\Phi\right) / \beta$ and find its minimum value $\hat{\mu}_{e T}$.

(f) For each discretized value $\beta_{j}$, use the Laguerre-Gauss quadrature method to calculate the integral term in Eq. (12), and calculate others on the left (ion) side and on the right (electron) side. Their relative difference, $\delta\left(\beta_{j}\right)=\left|1-n_{e}\left(\beta_{j}\right) / n_{i}\left(\beta_{j}\right)\right|$, is a measure of the remaining neutrality error at that location, $\beta_{j}$. The relative, rather than the absolute, error is used because of the strong decrease in the density along the expansion (several orders of magnitude).
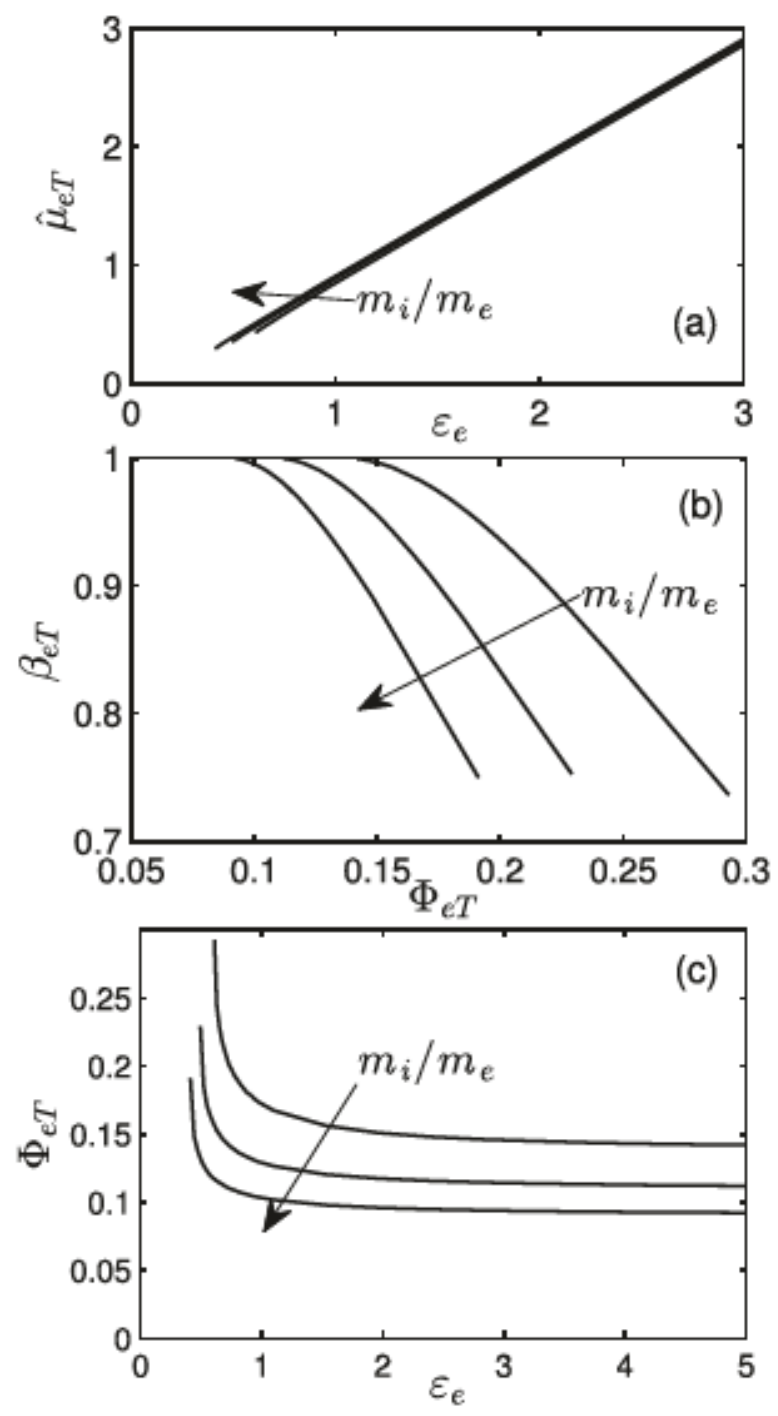

FIG. 3. Parametric analysis of (a) the minimum of the $\hat{\mu}_{e m}$ curves, $\hat{\mu}_{d \tau}\left(\varepsilon_{\varepsilon}\right)$, (b) the position of this minimum in terms of the solution $\beta_{e T}\left(\Phi_{T}\right)$, and (c) the relation between the electron energy ant the potential where the minimum is located $\Phi_{T}\left(\varepsilon_{\varepsilon}\right)$. Results are for the following values of the dimensionless parameters: $\varepsilon_{i}=0.1$ and $m_{i} / m_{c}=10^{3}, 10^{4}, 10^{5}$. Arrows indicate mass ratio increasing. 
(g) The net flux $\hat{\Gamma}=\hat{\Gamma}_{i}-\hat{\Gamma}_{e}$ is also computed from the guessed distribution $\Phi_{j}$. The current condition is incorporated through the $\gamma$ error parameter: $\gamma=\hat{\Gamma}-\hat{\Gamma}^{*}$, with $\hat{\Gamma}^{*}$ being the prescribed flux difference (zero in what follows).

(h) To quantify the overall error, compute the modified sum of squares,

$$
\Delta=\sum_{j} \delta^{2}\left(\beta_{j}\right)+m \gamma^{2},
$$

over the $\beta$ grid. Here, $m$ is a weighting factor chosen to balance the neutrality and current errors. A numerical optimization method is then used to minimize $\Delta$ over the set of trial functions $\Phi_{j}\left(\beta_{j}\right)$, plus the trial value of the dimensionless total potential drop $\Psi$. The numerical algorithm used is a trust region method based on interior point techniques, as described in Refs. 16 and 17. This method is very robust for solving the current
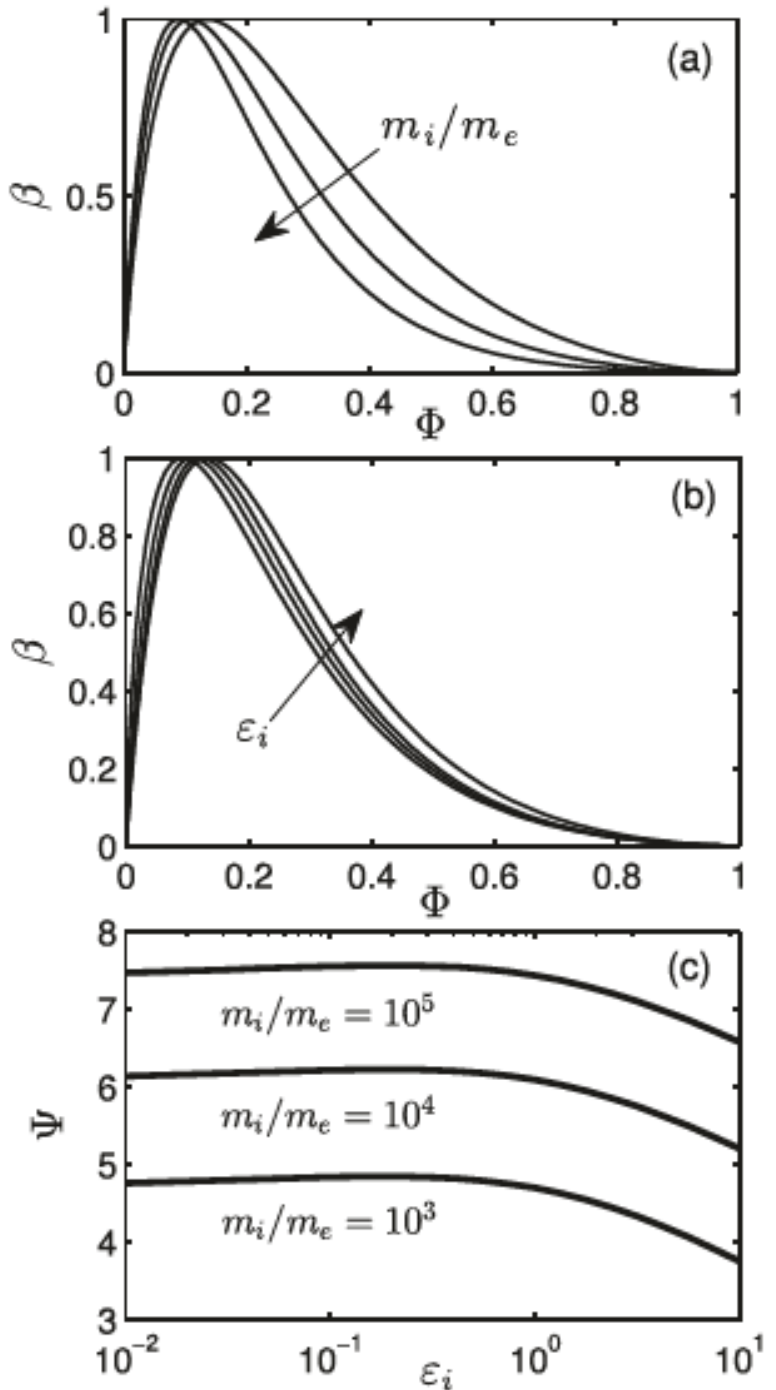

FIG. 4. (a) and (b) Dimensionless magnetic field as a function of nomalized electric potential, for several values of the mass ratio $m_{i} / m_{e}$ and the ion energy $\mathrm{g}_{i}$. In (a), the arrow indicates $m_{i} / m_{e}$ increasing $10^{3}, 10^{4}, 10^{5}$, at a constant $E_{i}=0.1$. In (b), the arrow indicates $E_{f}$ increasing, $(0.01,0.1,1,10)$ at a constant $m_{i} / m_{\epsilon}=10^{4}$. (c) depicts the total potential drop $\Psi=$ $-e \phi_{\text {oust }} / k T_{e \infty \infty}$ as a function of $\varepsilon_{i}$ and $m_{i} / m_{\varepsilon}$. problem, although this robustness penalizes somewhat the convergence rate.

(i) The above process is iterated until $\Delta \leq \epsilon$, with $\epsilon \ll 1$ the numerical tolerance desired for the solution.

\section{MOMENTS AND RESULTS}

Once the electric potential function $\Phi(\beta)$ and its total drop $\Psi$ are determined, it is possible to compute moments of the EDF and IDF (Eqs. 4 and 8 ) in order to compute macroscopic variables: ion and electron densities, fluxes, temperatures, heat fluxes, etc. The standard definitions for ions are listed in Eqs. (15) through (18):

$$
\begin{gathered}
\Gamma_{i}=n_{i} u_{i}=n_{i}\left\langle w_{i \|}\right\rangle, \\
k T_{i \|}=\left\langle m_{i} c_{i \|}^{2}\right\rangle, \text { with } c_{i \|}=w_{i \|}-u_{i},
\end{gathered}
$$
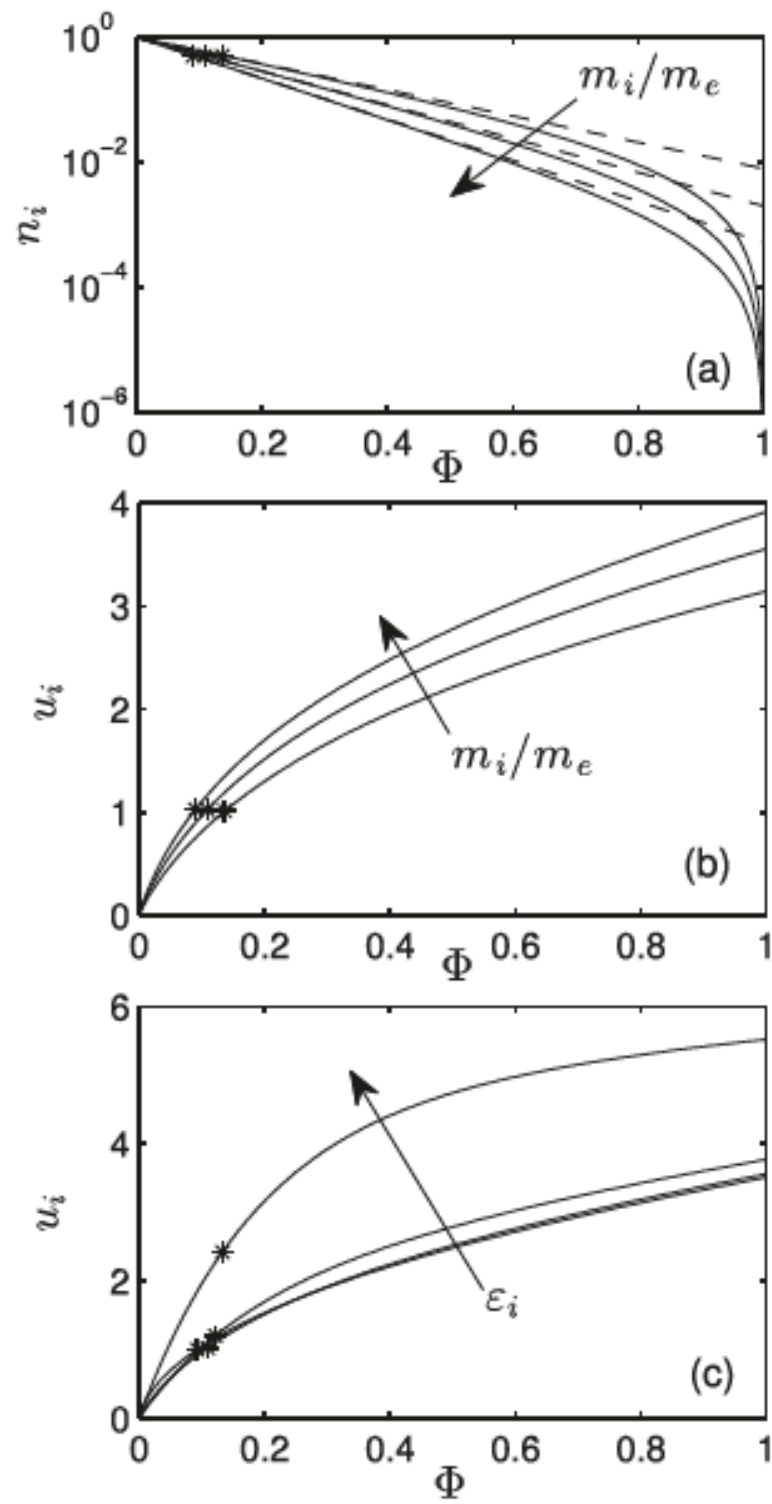

FIG. 5. (a) Dimensionless density $n_{i}(\Phi)=n_{e}(\Phi)$ at ion energy $\varepsilon_{l}=0.1$ for mass ratios $m_{i} / m_{e}=10^{3}, 10^{4}, 10^{3}$. Dashed lines show the Boltzmann equilibrium density for comparison, (b) and (c) Jon Mach number $u_{i}=u_{k}$ (for zero net current), using the same mass ratios and ion energies as in Figure 3. Hereafter, asterisks indicate the magnetic throat location $\Phi(\beta=1)$. 


$$
\begin{gathered}
k T_{i \perp}=\left\langle m_{i} w_{i \perp}^{2} / 2\right\rangle=B\left\langle\mu_{i}\right\rangle, \\
q_{i \|}=n_{i}\left\langle m_{i} c_{i \|}^{3} / 2\right\rangle ; \quad q_{i \perp}=n_{i}\left\langle m_{i} w_{i \perp}^{2} c_{i \|} / 2\right\rangle .
\end{gathered}
$$

The same expressions can be used for electrons by replacing subscripts $i$ by $e$. For presentation, ion and electron fluxes are normalized with $n_{\infty} c_{s}$ with $c_{s}=\sqrt{k T_{e \infty} / m_{i}}$, the ion sound speed. Temperature is normalized with $T_{i \infty}$ or $T_{e \infty}$ for ions and electrons respectively. The reference heat fluxes are $q_{i \infty}=n_{\infty} c_{s} k T_{i \infty \infty}$ and $q_{e \infty}=n_{\infty} c_{s} k T_{e \infty \infty}$.

For zero net current, the dimensionless variables that control the overall behavior of the plasma flow through the magnetic channel are the ion to electron mass ratio, $m_{i} / m_{e}$, and the ion energy to electron temperature ratio, $\varepsilon_{i}=E_{i \infty} / k T_{e \infty}$. Accordingly, we obtain the solution for the magnetic field distribution $\beta(\Phi)$ as a function of these two parameters. First, we present in Figure 3 the parametric behavior of $\hat{\mu}_{e T}\left(\varepsilon_{e}\right), \beta_{e T}\left(\Phi_{T}\right)$, and $\Phi_{T}\left(\varepsilon_{e}\right)$, which have been necessary for the computation of all moments.
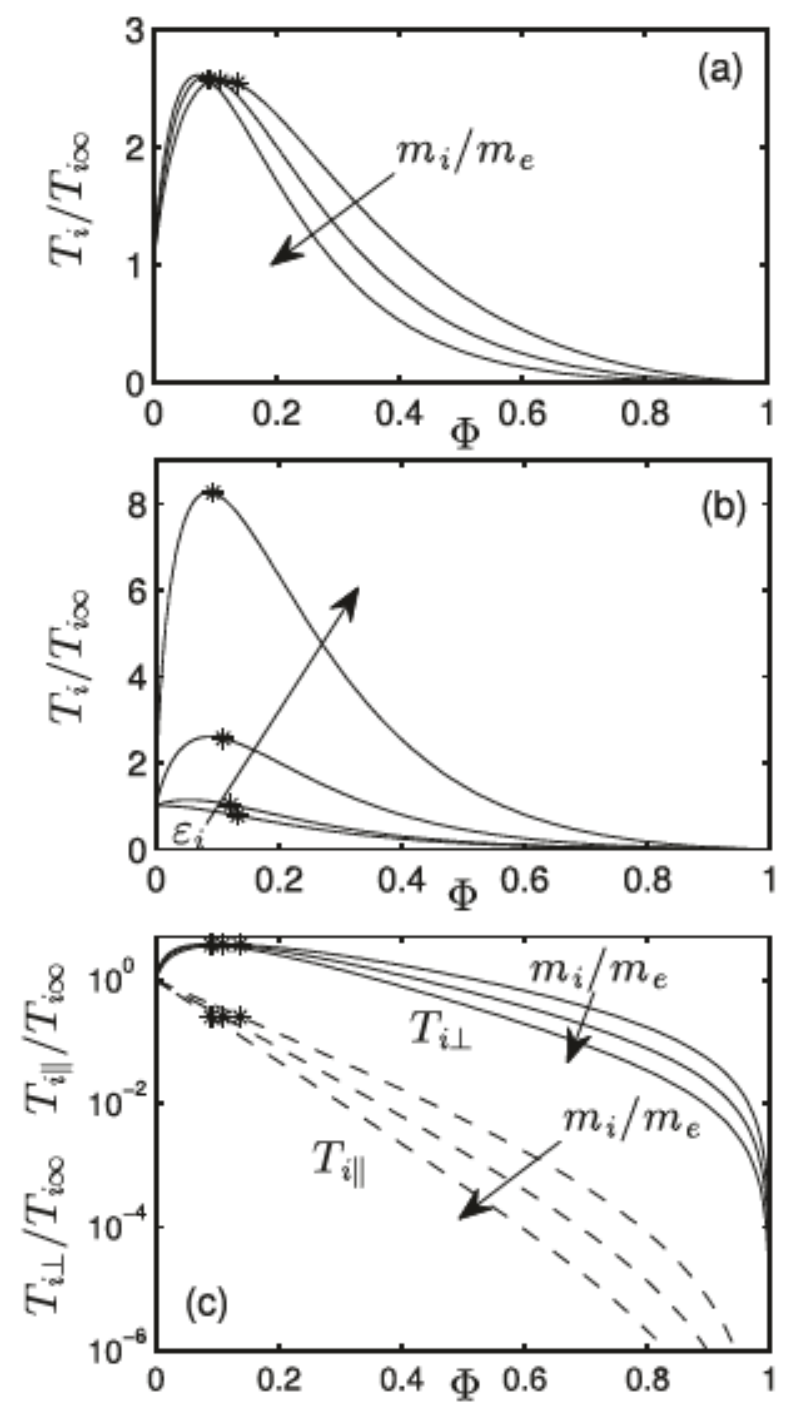

FIG. 6. (a) Ion total temperature $T_{i}=(2 / 3) T_{i \perp}+(1 / 3) T_{i \mid}$ as a function of the electric potential drop $\Phi$ (along the expansion), and the mass ratio $m_{i} / m_{e}=10^{3}, 10^{4}, 10^{5}$ for $B_{i}=0.1$. (b) The same quantity plotted in (a), but varying the ion energy factor $s_{i}=[0.01,0.1,1,10]$ for $m_{i} / m_{e}=10^{4}$. (c) Separate ion perpendicular and parallel temperatures $T_{i \perp}(\Phi), T_{i \rrbracket}(\Phi)$, for $\varepsilon_{i}=0.01$ and $m_{i} / m_{e}=10^{3}, 10^{4}, 10^{5}$.
The potential vs. magnetic field results are depicted in Figure 4 for the usual condition of zero net current. The total electric potential drop $\Psi$ increases for larger $m_{i} / m_{e}$. Note that this drop would vary if the net current condition was modified.

The density $n_{i}(\Phi)=n_{e}(\Phi)$ (0th moment) and the ion velocity $u_{i}=\left\langle w_{i \|}\right\rangle$, or "ion Mach number," are depicted in Figure 5. Since we also impose the zero-current condition, we have $u_{e}=\left\langle w_{e} \|\right\rangle=u_{i}$. Density is compared with the Boltzmann equilibrium prediction (dashed lines in Figure 5(a)),

$$
n_{i} / n_{\infty}=\exp \left(e \phi / k T_{e \infty}\right),
$$

showing that this law is accurate in the subsonic and moderately supersonic part of the flow, but loses its validity as one moves forward along the expansion.

Figures 6(a) and 6(b) show the total ion temperature variation $T_{i}=(2 / 3) T_{i \perp}+(1 / 3) T_{i \|}$ along the expansion for various mass ratios and ion energies. Figure 6(c) displays the parallel and perpendicular temperatures separately. From these results, we can conclude that ions become anisotropic through the expansion: the parallel temperature drops monotonically towards zero downstream, while the perpendicular temperature follows roughly the magnetic field variation, mimicking the magnetic moment conservation, and reaching its maximum close to $\beta=1$. An axial ion beam of zero temperature is formed downstream $\left(T_{i} \rightarrow 0\right.$ when $\Phi \rightarrow 1$ ). However, this is only true for the ion monoenergetic model considered here, which does not allow any velocity dispersion at the end of the expansion. The effect of an energy dispersion is seen below in the electron temperature (Figure 7).
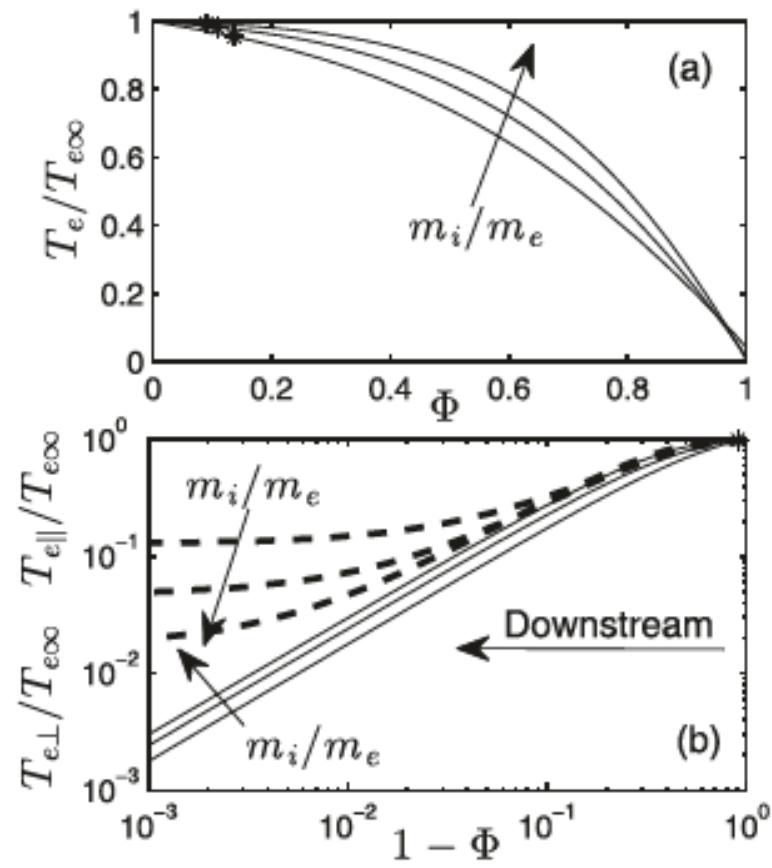

FIG. 7. (a) Total electron temperature $T_{e}(\Phi)$, for $m_{i} / m_{k}=10^{3}, 10^{4}, 10^{5}$ and $E_{i}=0.1$. (b) Electron perpendicular and parallel temperature-solid and dashed lines, respectively. All of them are for the same parameters in (a). The abscissa scale in (b) has been modified to emphasize the lower bound of $T_{\epsilon \mid}(\Phi)$ when $\Phi \rightarrow 1$. 
Figure 7 displays the electron temperature profile and points out the existence of electron collisionless cooling. This cooling response, as well as the loss of isotropy, would not be present if electrons were well confined. The perpendicular temperature $T_{e \perp}$ approaches zero as the magnetic field decreases and the potential approaches its limit, but the parallel component $T_{e \|}$ tends to a finite downstream limit. In this region, "hot" or energetic electrons control this and other variables, because lower energy electrons have been bounced back electrostatically. These "hot" electrons are also the ones that ensure the current-free condition. The electron cooling that occurs here is a consequence of the partial depletion of the EDF due to the electric potential barrier. This is also responsible for the loss of electron isotropy in the divergent side, with the perpendicular temperature becoming progressively smaller than the parallel temperature. It appears to be also responsible for the finite limit of the parallel electron temperature: the electrons that escape to infinity (those with $E_{e}>-e \phi_{\text {oud }}$ ) preserve the energy dispersion
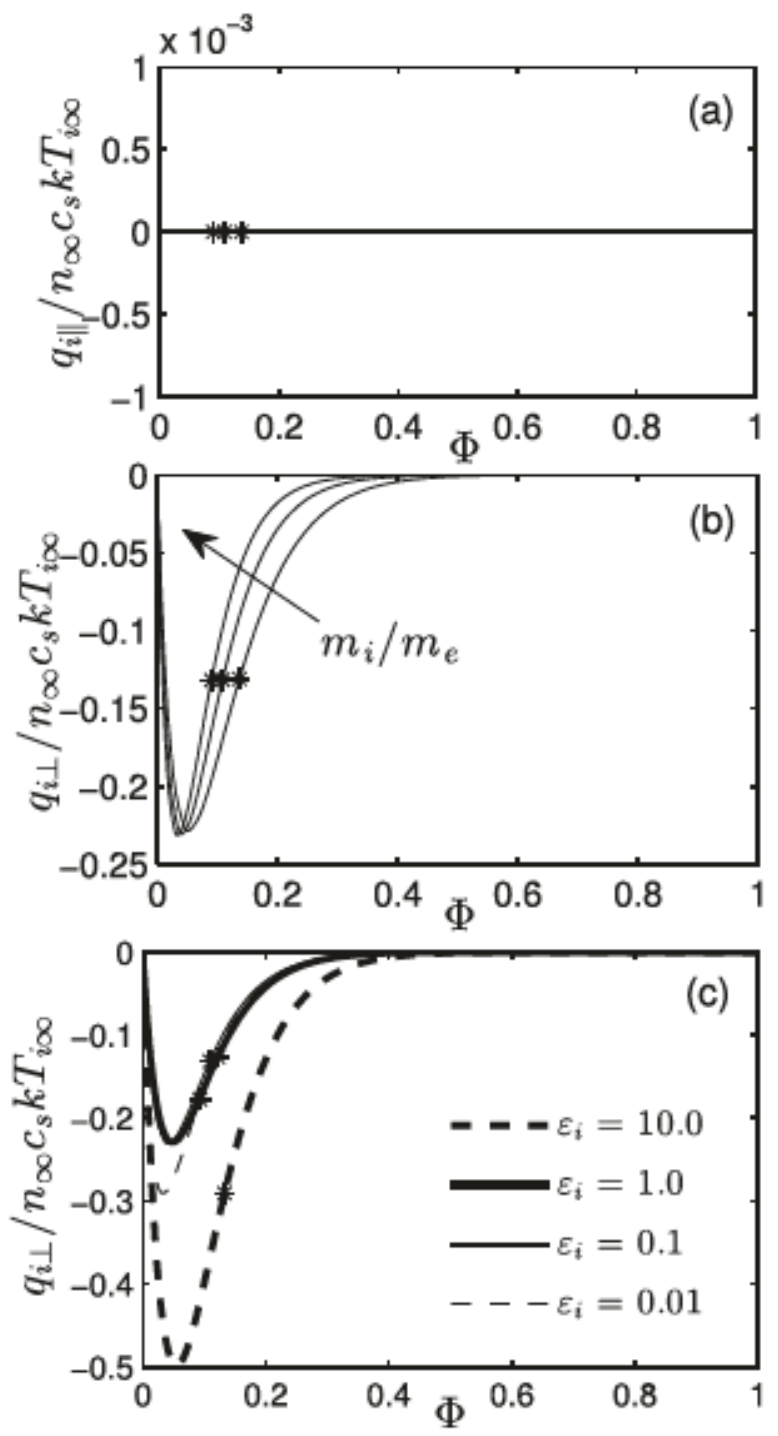

FIG. 8. Ion parallel (a) and perpendicular (b) and (c) heat fluxes. In (b), $\varepsilon_{i}=$ 0.1 and $m_{i} / m_{c}=10^{3}, 10^{4}, 10^{5}$. In (c), $\varepsilon_{i}=0.01,0.1,1,10$ and $m_{i} / m_{\epsilon}=10^{4}$. they had in the upstream reservoir. This lower bound on the electron temperature is quite dependent on the ion to electron mass ratio, and decreases as this mass ratio becomes larger (see Figure 7(b)).

The heat fluxes can be calculated by taking the 3rd moment of distribution functions, according to Eq. (18). For mono-energetic ions, the parallel heat flux can be seen analytically to be zero, $q_{i \|}=0$. This result is shown in Figure 8(a), and is very useful as a check of the consistency of the solution $\Phi(\beta)$. For its part, the random parallel flux of perpendicular energy $q_{i \perp}(\Phi)$ is negative everywhere along the expansion (see Figures 8(b), and 8(c)). Since we have seen that $T_{i \perp}(\Phi)$ decreases in the forward direction beyond the throat, the usual sign criterion of the Fourier heat transport law, $q=-K \partial_{x} T$, does not apply here, although it would in the convergent segment, as noted in Ref. 15 ( $K$ would be the thermal conductivity). It is interesting to speculate as to whether this partial heat flux with the same sign as the corresponding temperature gradient violates the 2 nd law of thermodynamics. Presumably, this is not so because it occurs only for one of the components of the heat flux and of the temperature of the whole fluid.

Finally, regarding electron heat fluxes, the parallel $q_{e \|}$ and perpendicular $q_{e \perp}$ contributions are presented in Figure 9. Although the plasma is treated as collision-less, $q_{e \|}(\Phi)$ agrees with the sign criterion of the Fourier law. Zero heat fluxes are obtained at both limits, infinitely upstream and infinitely downstream, $q_{e \|} \rightarrow 0$ when $\Phi \rightarrow 1$. The sharp maximum of the parallel component on the convergent region, and the peak of the perpendicular one just at the throat position, cannot be satisfactorily explained by these authors.

\section{DISCUSSION}

Because of the assumed presence of the trapped electron population, the influence of free electrons on the
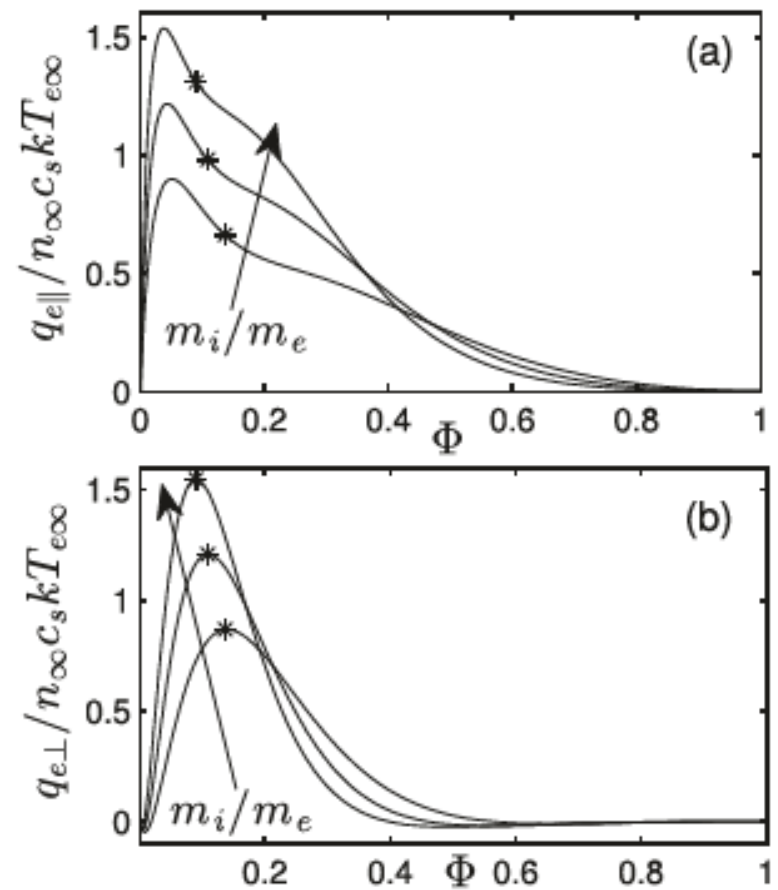

FIG. 9. Parallel (a) and perpendicular (b) electron heat fluxes, $s_{i}=0.1$ and $m_{l} / m_{e}=10^{3}, 10^{4}, 10^{5}$. 
obtained results tums out to be marginal only. The exception, of course, is the odd moments i.e. mean velocity, for which these free electrons are, in fact, the only contributors. If the depletion of the trapped population was possible, or, more generally, if the trapping mechanism imposed a distribution function different from that assumed here, a different shape of the solution $\Phi(\beta)$ for the relationship between electric potential and magnetic field would be obtained and it is difficult to predict how the results presented here would be modified. We can, however, anticipate that a reduction in the trapped population would cause an earlier degradation of the Maxwell-Boltzmann equilibrium along the magnetic channel, because the influence of free-electrons on the computed electron density would be stronger and their effects would be manifested before reaching the far field of the plasma expansion. Similarly, there would be a strong impact on the electron temperature, presumably resulting in less isotropy than the results presented here, which are dominated by the isotropic confined group.

All macroscopic parameters are computed according to the local distribution function of both ions and electrons. And, this is determined self-consistently by the solution
$\Phi(\beta)$. To better illustrate the evolution of the distribution function along the magnetic channel, Figure 10 depicts $g=$ $g\left(w_{e \|}, w_{e \perp}\right)$ at several positions on the convergent side (labeled by $\Phi=0.004,0.02,0.06$ ), magnetic throat (that corresponds to $\Phi \simeq 0.1$ ), and on the divergent side (labeled by $\Phi=0.5,0.9$ ), for the nominal case $m_{i} / m_{e}=10^{4}, \varepsilon_{i}=0.1$. These plots point out several effects already mentioned: the loss cone or the beam formation is clearly identified, and this justifies the loss of isotropy. Also, it is shown that even far downstream $(\Phi=0.9)$, a confined (trapped or bounced to the source) electron population is still there, preserving its isotropy. The depletion of the distribution function, in terms of energies, exerted by the effective potential $\mu_{e} B-e \phi$, is observable by the shorter peak of the $g=g\left(w_{e \|}, w_{e \perp}\right)=g(E)$ surfaces.

Our model assumes highly magnetized ions and electrons. It is conjectured that most of the features uncovered must be common to weakly magnetized or non-magnetized vacuum expansions, although, except for numerical simulations, ${ }^{3}$ this has not been explored in detail. One avenue for extending the work in that direction is the idea of an electrostatic invariant that was broached in our earlier work on cusp flows. ${ }^{15}$ In that case, ions were seen to be funneled into the cusp
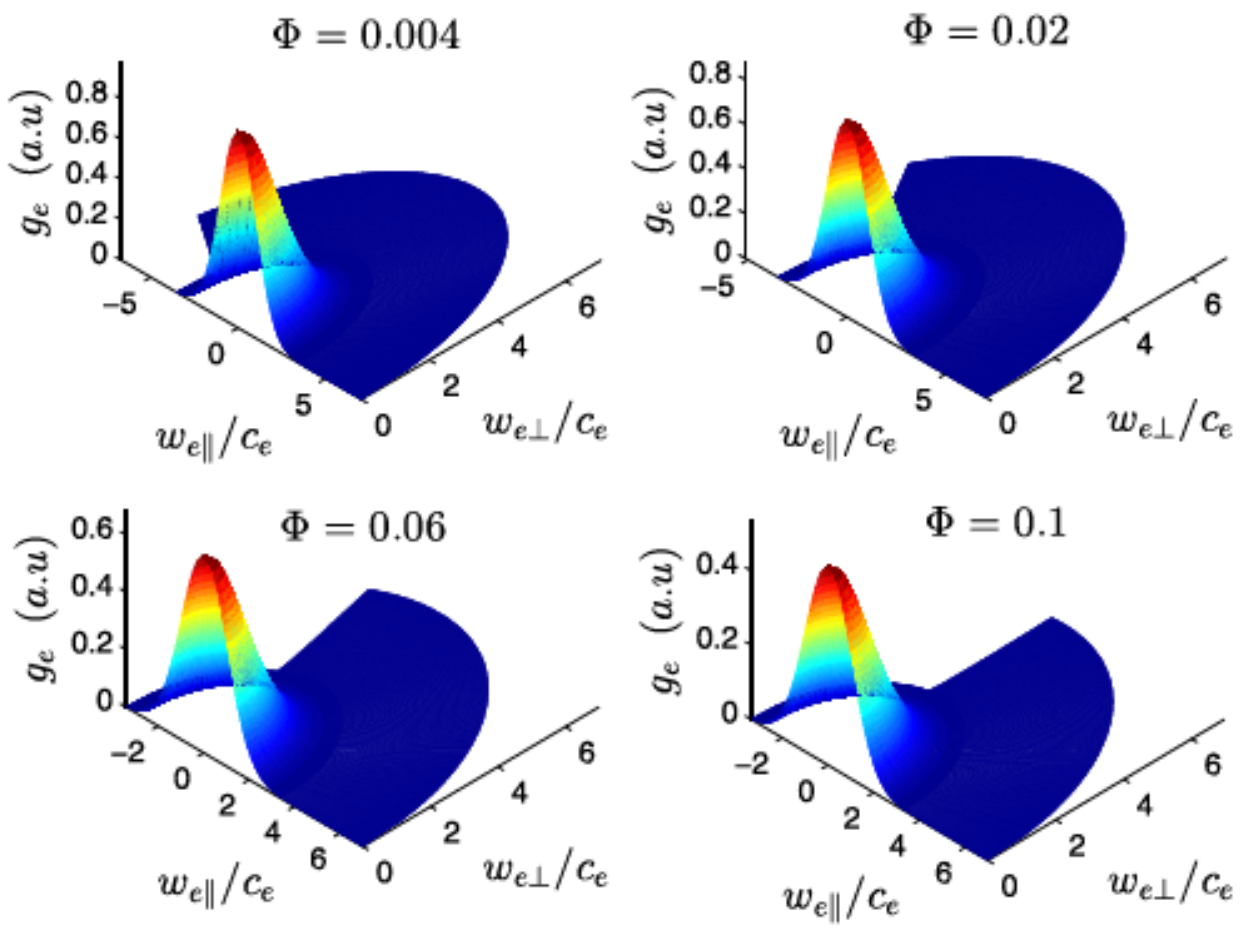

FIG. 10. Electron distribution function $g=g\left(w_{\perp}, w_{1}\right)$ at different locations: convergent side $(\Phi=0.004,0.02$, 0.06 , that corresponds to $\beta=0.1,0.5$, 0.9 , respectively), throat (or $\Phi=0.1$ ) and divergent side $(\Phi=0.5,0.9)$. Electron speed is dimensionless with the electron thermal speed $c_{\epsilon}=\sqrt{k T_{\epsilon \infty o} / m_{e}}$.
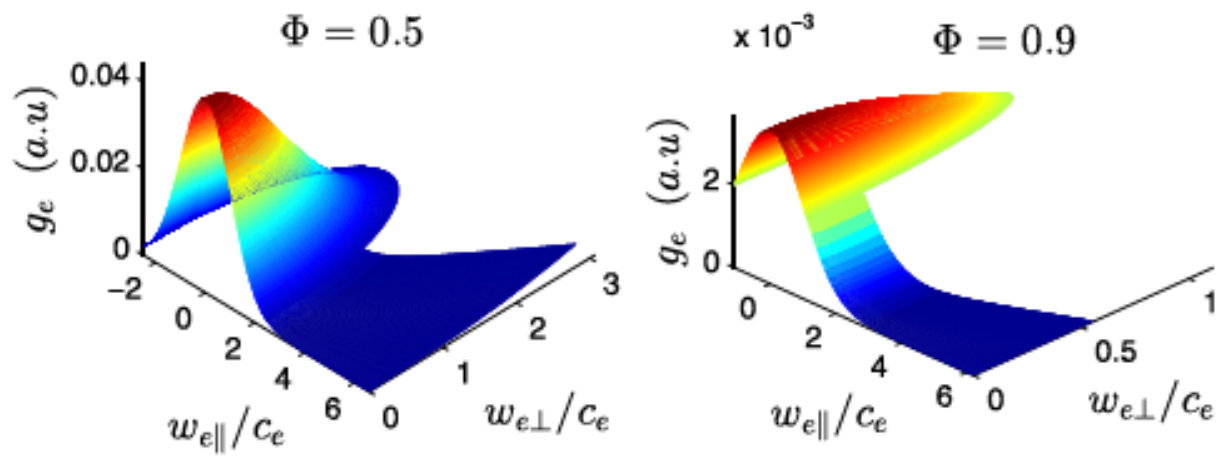


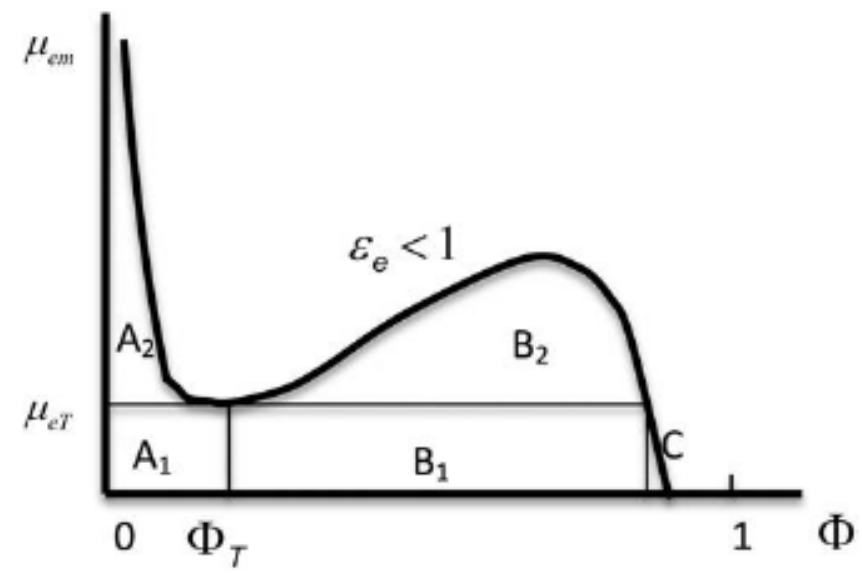

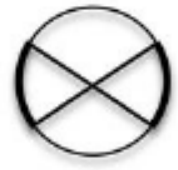

$A_{1}$

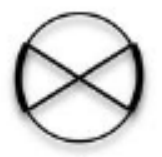

$B_{1}$

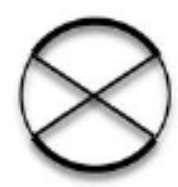

$\mathrm{A}_{2}$

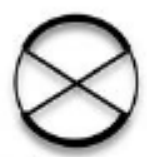

$\mathrm{B}_{2}$ (with trapping)

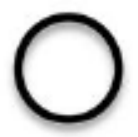

C (full sphere)

FIG. 11. Angular electron distribution function for $\varepsilon_{\varepsilon}^{*}<\varepsilon_{\varepsilon}<1$.

by the formation of a self-consistent potential well due to the magnetically guided electrons. For our present purposes, in the absence of magnetization, electrons are radially confined by the potential hump forced by the inertial concentration of ions near the axis, and a similar approach appears to be possible.

We assume here a magnetic field distribution and shape that is separately determined by coils or magnets. Under high magnetic Reynolds number conditions, as in astrophysical jets, the magnetic field is in fact a self-consistent part of the solution. However, since our results are parameterized with $B$ regardless of its spatial distribution, they remain valid in any case.

\section{CONCLUSIONS}

For magnetized, collisionless ions and electrons, steady solutions have been found for the expansion to vacuum of a fully neutralized plasma jet with zero net current. The electron density over most of the jet is dominated by confined electrons. On the divergent side, this is controlled mainly by the subgroup of trapped electrons that are isolated electrostatically from the downstream environment and magnetically from the upstream chamber. The small fraction that escapes provides the necessary electron flux to keep a current-free plasma beam, a mandatory condition for all electrodeless thrusters mentioned in the introduction. Ion and electron distributions preserve their isotropy only over restricted angular ranges at each energy; as a consequence,

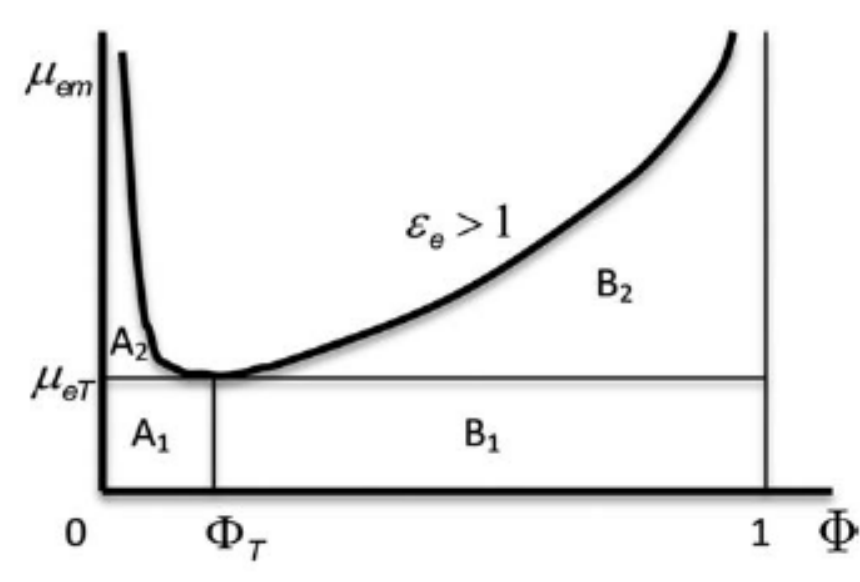

$\mathrm{A}_{1}$

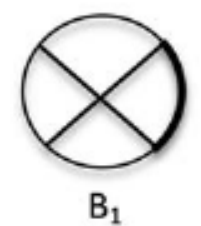

$\mathrm{B}_{1}$

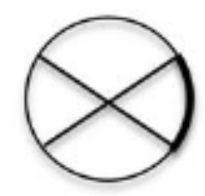

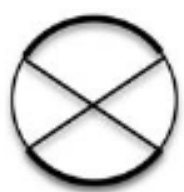

$\mathrm{A}_{2}$

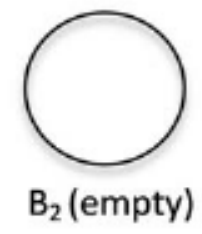

FIG. 12. Angular electron distribution function for $\varepsilon_{e}>1$.

heat fluxes develop despite the absence of collisions. In addition, whole electron energy ranges are empty or only partially populated, and this leads to reductions of the various temperatures towards the exit, causing the electron cooling phenomenon. The total potential drop between reservoir and exit conditions is calculated as part of the self-consistent solution, and it scales with the electron/ion mass ratio in a similar fashion to the known sheath potential drop. This is a very important result because this bounded drop (finite acceleration of ions) is more reasonable than the unbounded results predicted by isothermal or Maxwell-Boltzmann expansion models, or the unjustified adiabatic-polytrophic models used extensively in the literature. This finite drop is also responsible of the kinetic electron cooling effect.

\section{ACKNOWLEDGMENTS}

The research leading to these results has been funded by the Spanish R\&D National Plan (Grant No. ESP2013-41052P). Navarro-Cavallé acknowledges also support from the FPU fellowship program of the R\&D National Plan (Grant No. AP2010-0791).

\section{APPENDIX: ANGULAR ELECTRON DISTRIBUTION FOR VARIOUS ENERGY RANGES}

In this Appendix, the electron velocity distribution function is discussed, in terms of the angle formed by the velocity vector and the parallel direction. The behavior depends on the electron energy, defining the following three ranges: 
(a) For $\varepsilon_{e}<\varepsilon_{e}^{*}$, the distribution is a full spherical shell.

(b) For $\varepsilon_{e}^{*}<\varepsilon_{e}<1$, the shape of the distribution function depends also on $\mu_{e}\left(\varepsilon_{e}, \Phi\right)$ and $\mu_{e T}\left(\varepsilon_{e}\right)$. Figure 11 schematically details this dependence.

(c) For $\varepsilon_{e}>1$, the shape is depicted in Figure 12.

${ }^{1}$ O. Buneman, J. Nucl, Energy C 2(1), 119-134 (1961).

${ }^{2}$ H. Derfler, Phys. Fluids 7(10,) 1625-1637 (1964).

${ }^{3}$ J. Wang, O. Chang, and Y. Cao, IEEE Trans. Plasma Sci. 40(2), 230-236 (2012).

${ }^{4}$ M. Merino and E. Ahedo, IEEE Trans. Plasma Sci. 43(1), 244-251 (2015).

${ }^{5}$ M. W. Liemohn and G. V. Khazanov, Phys. Plasmas 5(3), 580-589 (1998).

${ }^{6}$ A. V. Arefiev and B. N. Breizman, Phys. Plasmas 16, 055707 (2009).

${ }^{7}$ C. Charles and R. Boswell, Appl. Phys. Lett. 82(9), 1356-1358 (2003).

${ }^{8} \mathrm{O}$. Batishchev, "Minihelicon plasma thruster," IEEE Trans. Plasma Sci. 37(8), 1563-1571 (2009).
'D. Pavarin, F. Ferri, M. Manente, D. Curreli, Y. Güclü, D. Melazzi, D. Rondini, S. Suman, J. Carlsson, C. Bramanti, E. Ahedo, V. Lancelotti, K. Katsonis, and G. Markelov, in 31 st International Electric Propulsion Conference (IEPC, 2009), p. 205.

${ }^{10} \mathrm{~J}$. M. Little and E. Y. Choueiri, Phys. Plasmas 20, 103501 (2013).

${ }^{11}$ E. Ahedo and J. Navarro-Cavallé, Phys. Plasmas 20(4), 043512 (2013).

${ }^{12}$ F. R. Chang-Díaz, J. P. Squire, R. Bengtson, B. Breizman, M. D. Carter, and F. Baity, "The Physics and Engineering of the VASIMR Engine," AIAA Paper 2000-3756, 2000.

${ }^{13}$ B. W. Longmier, E. A. Bering III, M. D. Carter, L. D. Cassady, W. J. Chancery, F. R. Chang-Díaz, T. W. Glover, N. Hershkowitz, A. V. Ilin, G. E. McCaskill, C. S. Olsen, and J. P. Squire, Plasma Sources Sci. Technol. 20(1), 015007 (2011).

${ }^{14}$ J. Jarrige, P. Q. Elias, F. Cannat, and D. Packan, "Characterization of a coaxial ECR plasma thruster," AIAA Paper 2013-2628, 2013.

${ }^{15} \mathrm{M}$. Martinez-Sanchez and E. Ahedo, Phys. Plasmas 18(3), 033509 (2011).

${ }^{16}$ R. H. Byrd, J. C. Gilbert, and J. Nocedal, Math. Program. 89(1) 149-185 (2000).

${ }^{17}$ R. A. Waltz, J. L. Morales, J. Nocedal, and D. Orban, Math. Programm. 107(3), 391-408 (2006). 